\title{
The blood cerebrospinal fluid barrier orchestrates immunosurveillance, immunoprotection, and immunopathology in the central nervous system
}

\author{
Maria Ayub ${ }^{1,2,3}$, Hee Kyung Jin $^{1,4}$ E Jae-sung Bae $e^{1,2,3, *}$ \\ ${ }^{1}$ KNU Alzheimer's disease Research Institute, Kyungpook National University, Daegu 41566, ${ }^{2}$ Department of Physiology, School of \\ Medicine, Kyungpook National University, Daegu 41944, ${ }^{3}$ Department of Biomedical Science, BK21 Plus KNU Biomedical Convergence \\ Program, Kyungpook National University, Daegu 41944, ${ }^{4}$ Department of Laboratory Animal Medicine, College of Veterinary Medicine, \\ Kyungpook National University, Daegu 41566, Korea
}

\begin{abstract}
Once characterized as an immune privileged area, recent scientific advances have demonstrated that the central nervous system (CNS) is both immunologically active and a specialized site. The anatomical and cellular features of the brain barriers, the glia limitans, and other superficial coverings of the CNS endow the brain with specificity for immune cell entry and other macro- and micro-elements to the brain. Cellular trafficking via barriers comprised of tightly junctioned non-fenestrated endothelium or tightly regulated fenestrated epithelium results in different phenotypic and cellular changes in the brain, that is, inflammatory versus regulatory changes. Based on emerging evidence, we described the unique ability of the blood cerebrospinal fluid barrier (BCSFB) to recruit, skew, and suppress immune cells. Additionally, we sum up the current knowledge on both cellular and molecular mechanisms governed by the choroid plexus and the cerebrospinal fluid at the BCSFB for immunosurveillance, immunoprotection, and immunopathology. [BMB Reports 2021; 54(4): 196-202]
\end{abstract}

\section{INTRODUCTION}

Prior allograft studies of tissue and tumor transplantation into the brain parenchyma have described the successful growth of heterogeneous tumors and their respective tissues. Given the brain's tolerance of these transplants and the frequent rejections at the periphery, the brain was proposed to be a immunoprivileged system (1). Immunoprivilege has formerly been con-

*Corresponding author. Tel: +82-53-420-4815; Fax: +82-53-424-3349; E-mail: jsbae@knu.ac.kr

https://doi.org/10.5483/BMBRep.2021.54.4.205

Received 25 September 2020, Revised 10 October 2020, Accepted 23 November 2020

Keywords: Brain barriers, Choroid plexus, Immunoregulation, Neuroimmune communication sidered to be a neuroprotective mechanism that shields the central nervous system (CNS) from undulating peripheral cues and was suggested to be immutable, whereas recent findings argue that CNS is in a continuously dynamic state (2). For instance, modern in vivo imaging studies have exhibited previously undiscovered glymphatic and lymphatic systems which are cerebrospinal fluid (CSF) and interstitial fluid drainage networks, working as waste removal and communicators between CNS and periphery and maintain CNS immune homeostasis $(3,4)$.

Similarly, the idea of brain barriers (blood-brain barrier (BBB), blood leptomeningeal barrier (BLMB), and blood cerebrospinal fluid barrier (BCSFB)) that function as checkpoints for immune cells as well as other peripheral components that traffic to the CNS have urged the scientific community to revisit the concept of CNS immunoprivilege. Here we evaluate both the anatomical localization and physiological functions of the CNS barriers, focusing on the BCSFB role in CNS immunoregulation and discuss the suitability of the BCSFB as a therapeutic target in various neurological disorders.

\section{CNS GATING SYSTEM: ABSOLUTE AND REGULATORY BARRIERS}

Considering the importance of CNS anatomy and its relevance in neuroimmune communication, the brain barriers are generally categorized into true and educational gates. Owing to their tightly junctioned endothelial cells (ECs), both the BBB and BLMB directly separate the circulating blood from the surroundings of the CNS so called absolute or true barriers. Conversely, the BCSFB, constituted of firmly placed epithelial cells of the choroid plexus (ChP), regulates the peripheral trafficking and is thus called an educational gate or immunoregulatory barrier.

\section{Absolute or true barriers}

The BBB is a far-reaching barrier of the CNS composed of a monolayer of non-fenestrated ECs held together by tight junc- 
tion transmembrane proteins. Attributed to its anatomical contribution, the BBB has a very potent role in prohibiting the immune cells infiltration via sonic hedgehog $(\mathrm{SHH})$ and $\mathrm{CXCL} 12$ expressed on its endothelium (Fig. 1A) $(5,6)$. However, under inflammatory conditions, the BBB facilitates cellular intrusion into the CNS, by upregulating the secretion of CCL2 which attracts $\mathrm{CD} 14^{+}$pro-inflammatory monocytes. The transforming growth factor $\beta$ (TGF $\beta$ ) and granulocyte-macrophage colonystimulating factor then differentiate the recruited cells into dendritic cells (DCs) (7). The BLMB is composed of the dura, arachnoid mater, subarachnoid space, and pia mater, which together are called the leptomeninges. These three layers together with subarachnoid space provide structural support, cushion, route for CSF circulation, and a barrier to the CNS (Fig. 1B) (8). The BLMB has been identified as a major site of pro-inflammatory leukocyte entry to the CNS during experimental autoimmune encephalomyelitis (EAE) (9) while in the normal state, negligible leukocyte transport is seen through this structure due to its restrictive nature. In a nutshell, BBB and $B L M B$ are true barriers that have no immunoregulatory properties and these findings support the notion that these barriers should remain sealed.

\section{Educational barrier or Immunoregulatory barrier}

The BCSFB, in contrast to the BBB and BLMB, functions as a controlled gate for neuroimmune communication as well as immunosurveillance. The BCSFB is a biophysical, biochemical, and buoyant barrier that provides a physical barrier to peripheral transfer, facilitates the removal of CNS wastes, and acts as an immunosurveillance system (10). The BCSFB is composed of the ChP and CSF. The ChP consists of monolayered cuboidal epithelial cells lined together by tight junctions, floating in the brain ventricles (Fig. 1C). The most extensively known function of the ChP is CSF production; thus, the ChP-CSF system has been named. ChP-CSF system dispenses crucial health and growth-promoting signals in the CNS. Disruptions of ChP-CSF system have been observed in a range of neurological diseases such as hydrocephalus, ChP tumors, Alzheimer's disease (AD), and the loss of neuroregenerative capabilities (11).

\section{IMMUNOSURVEILLANCE, IMMUNOPROTECTION, AND IMMUNOPATHOLOGY AT THE BCSFB}

Due to the presence of specialized CNS barriers, immune cell entry into the CNS parenchyma is a two-step process: [1] migration of immune cells across the BBB or BCSFB and [2] their rolling over across the glia limitans into the CNS parenchyma. The distinctive anatomy of the BCSFB orchestrates the first step of this migratory process by regulating the expression of adhesion molecules, chemokines, and cytokines at the ChP and in the CSF and thus mediates immunosurveillance, immunoprotection, and immunopathology in the CNS (Fig. 2).

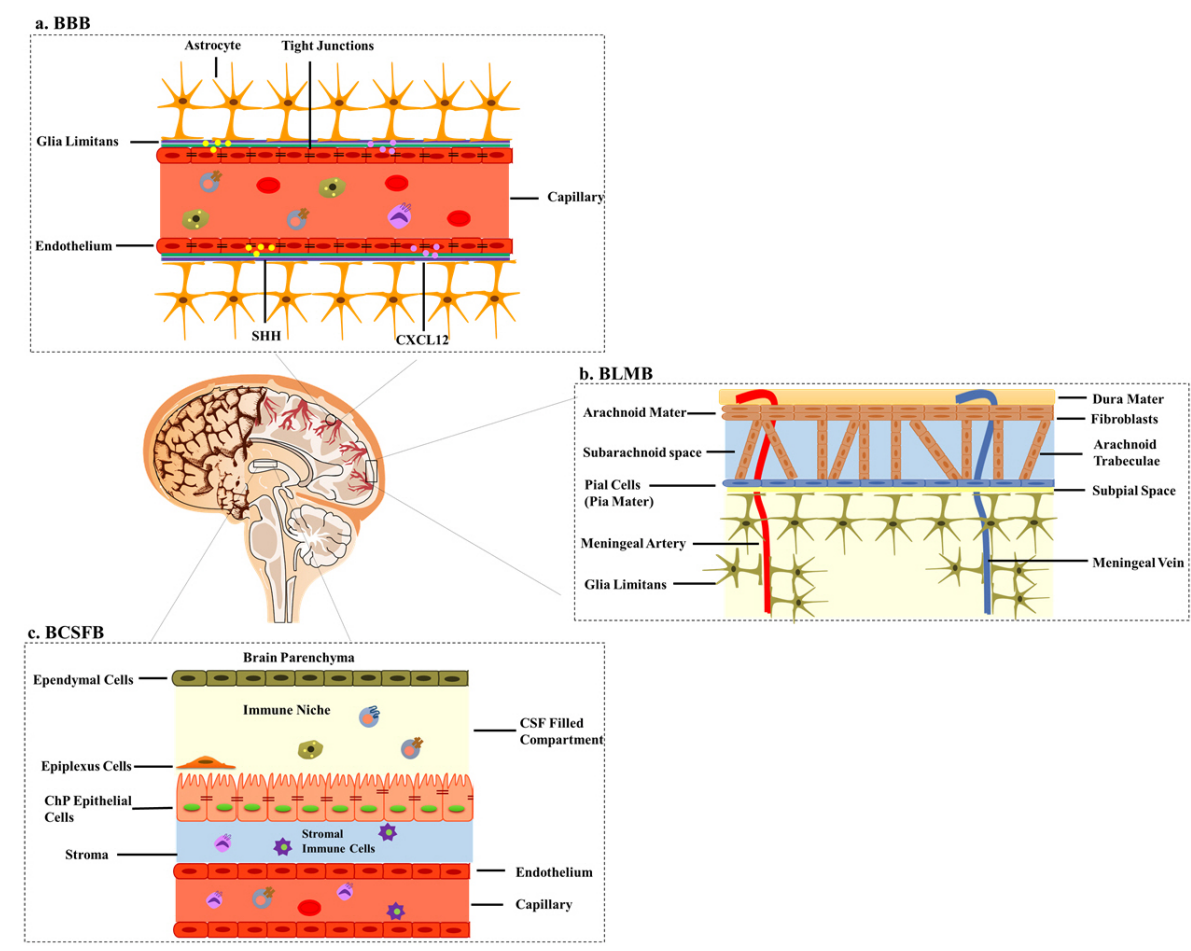

http://bmbreports.org
Fig. 1. CNS barriers. (A) The BBB is composed of endothelial cells with tight junctions fenced by the glia limitans, contributing to the true nature of this system. SHH: sonic hedgehog. (B) The BLMB is composed of the dura mater, the arachnoid mater, subarachnoid space, and the innermost layer pia mater. (C) The BCSFB, which is composed of the $\mathrm{ChP}$ and CSF, lies in the brain ventricles and has its own CSF and stromal immune cell niche. 

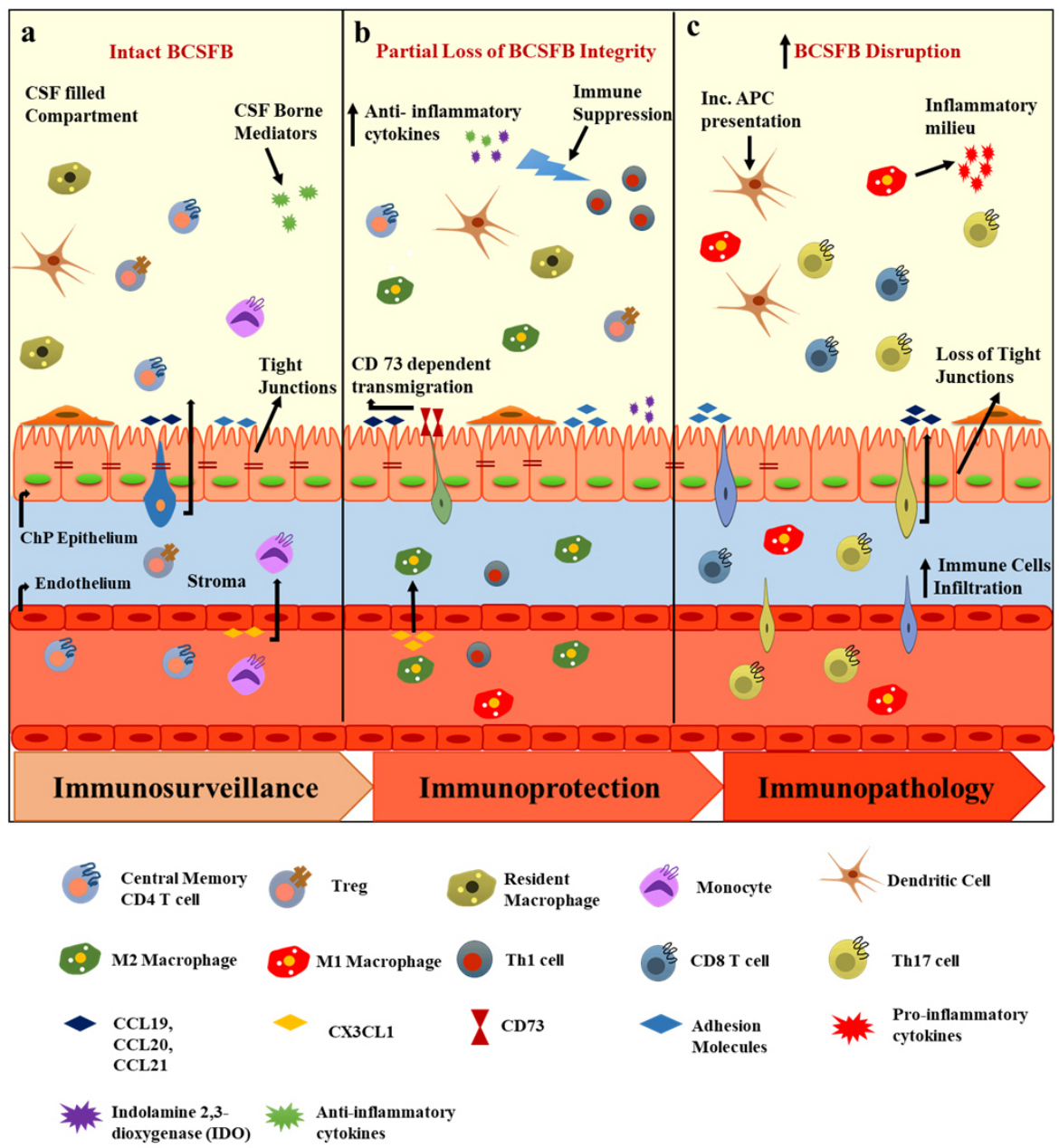

Fig. 2. Immunoregulation at the BCSFB. (A) In the homeostatic state, the BCSFB is populated with $T_{C M}$ and $T_{E M}$ cells. The ChP expresses chemokines which together with CSF-borne mediators (antiinflammatory molecules), participate in immunosurveillance (B) Following inflammatory stimuli or a brain injury, the integrity of the BCSFB is partially lost and immune selectivity and immune suppression are observed, which involve M2 macrophage transmigration at the $\mathrm{ChP}$ and the suppression of IFN $\gamma$-producing Th1 cells by CSF. (C) In disease conditions, such as MS and AD, the BCSFB is disrupted, tight junctions are lost, resident antigen presentation increases, the CSF anti-inflammatory milieu shifts to the pro-inflammatory phenotype, and $\mathrm{CD}^{+}$and Th17 cells infiltration is enhanced.

\section{Differential expression of adhesion molecules and chemokines at the $\mathrm{ChP}$}

Adhesion molecules (such as cell adhesion molecules, selectins, cadherin, and other mediators) are vital to some processes of the immune cell response, namely, migration, interaction, and execution (12). A well-known immunoregulatory feature of the $\mathrm{ChP}$ is the continuous expression of these adhesion molecules on its epithelium and endothelium and their secretion into the CSF. Figarella-Branger et al. (13) have described that the expression of E-cadherin was exclusively localized to the basolateral membrane of ChP epithelial cells in healthy adult ChPs and ChP papillomas. Similarly, vascular cell adhesion molecule-1 (VCAM-1) and intercellular adhesion molecule-1 (ICAM-1) are expressed on the apical border of the ChP. Besides, VCAM-1, ICAM-1, and mucosal addressin cell adhesion molecule-1 (MAdCAM-1) are upregulated during EAE (14). The CSF of healthy individuals is populated with $\mathrm{CD} 4^{+}$memory $\mathrm{T}$ cells, which are observed to enter CNS through P-selectin-expressing endothelial cells of the ChP (15). As these studies show, the choroidal structure expresses factors that are central to the transport of peripheral cellular components into the CNS through the BCSFB.

Similarly, in the adult CNS, the BCSFB and BBB vasculatures express many homeostatic chemokines and their receptors (including CCL19-21, CCR6, CCR7, and CXCL12) and govern immunosurveillance, neuro- and glio-genesis, neuronal survival, and synaptic transmission (Fig. 2A).

CCL20-CCR6 axis: C-C chemokine receptor type 6 (CCR6) is expressed in lymphatic and non-lymphatic tissues, leukocytes, DCs, and natural killer cells $(16,17)$. CCL20, the only ligand for CCR6, is constitutively expressed on the ChP epithelium of both mice and humans under homeostatic conditions and is involved in the migration of interleukin-17 producing T-helper cells (Th17) during EAE (18). CCL20 is also expressed in the CSF as an early marker of pneumococcal meningitis, in which CCL20 alleviate the disease by attracting peripheral granulocytes and lymphocytes to the subarachnoid space (19). 
CCL19-CCL21-CCR7 axis: C-C chemokine receptor type 7 (CCR7) is expressed on a variety of immune cells including DCs, B cells, and T cells. Under homeostatic conditions, the CCR7 ligands CCL19 and CC21 are continually expressed in the CSF and by $\operatorname{ChP}(20,21)$. CCL19 and CCL21 are the only known ligands for CCR7 and under normal conditions they direct the migration of central memory $\mathrm{CD}^{+}{ }^{+} \mathrm{T}$ cells $\left(\mathrm{T}_{\mathrm{CM}}\right)$, which express CCR7 on their surface, into the CNS via the ChP.

$\mathrm{CX}_{3} \mathrm{CR} 1-\mathrm{CX}_{3} \mathrm{CL} 1$ signaling: $\mathrm{CX}_{3} \mathrm{C}$ chemokine receptor $1\left(\mathrm{CX}_{3} \mathrm{CR} 1\right.$, also known as the fractalkine receptor) and its ligand $\mathrm{CX}_{3} \mathrm{CL} 1$ (neuroactin) are extensively expressed in immune cells and monitor the adhesion and migration of immune cells (22). Under non-inflammatory conditions, $\mathrm{CX}_{3} \mathrm{CL} 1$ and the $\mathrm{CX}_{3} \mathrm{CR} 1-\mathrm{CX}_{3} \mathrm{CL} 1$ signaling system are highly expressed in ChP attachments, which regulate the peripheral $\mathrm{CX}_{3} \mathrm{CR} 1$-derived monocyte population in the $\mathrm{ChP}$ (23). Likewise, following spinal cord injury and stroke, the recruitment of $\mathrm{CX}_{3} \mathrm{CR} 1^{\mathrm{hi}} \mathrm{LY} 6 \mathrm{C}^{\mathrm{low}}$ monocyte-derived macrophages (M2) instead of $\mathrm{CX}_{3} \mathrm{CR} 1^{\text {low }} \mathrm{LY}_{6} \mathrm{C}^{\text {high }}$ monocyte-derived macrophages (M1) is seen via the $\operatorname{ChP}(24,25)$. Thus, the $\mathrm{CX}_{3} \mathrm{CR} 1-\mathrm{CX}_{3} \mathrm{CL} 1$ axis is essential for the cellular trafficking and immunoselective properties of the ChP. Studies of these adhesion molecules and chemokines expressed on the ChP epithelium and endothelium indicate the immunoregulatory properties of the ChP-CSF system.

\section{The IFN $\gamma$-IL-4-ChP axis}

The IFN $\gamma$-IL4-ChP system helps to recruit peripheral cells to the CNS by mediating the expression of chemokines and adhesion molecules at this site, thus regulating CNS homeostasis. In an assessment of porcine $\mathrm{ChP}$ treated with pro-inflammatory cytokines following Streptococcus suis induction, IFN $\gamma$ dependent ChP upregulation of indolamine 2,3-dioxygenase (IDO) was observed. IDO is a potent enzyme that degrades L-tryptophan (an amino acid essential for T-cell proliferation) and reduces the production of nitric oxide. The ChP is believed to exert bacteriostatic effects and initiates rescue from meningitis by regulating the IFN $\gamma$-IDO pathway of T-cell exhaustion (Fig. 2B) (26). Similarly, EAE studies of IFN $\gamma \mathrm{R}^{\mathrm{CNS}} \mathrm{KO}$ (CNS IFN $\gamma \mathrm{R}$ deletion) and IFN $\gamma \mathrm{R}^{\text {peri }} \mathrm{KO}$ (peripheral IFN $\gamma \mathrm{R}$ deletion) have indicated that IFN $\gamma R^{C N S}$ KO EAE mice had more severe and atypical neurological deficits than IFN $\gamma \mathrm{R}^{\text {peri }} \mathrm{KO}$ mice; lower levels of immune infiltration, enhanced microglia-induced neurotoxic damage, and disrupted neuronal microtubule network (27). Further, physiological aging influences IFN $\gamma$ regulation of IL-4 i.e., reduced levels of IFN $\gamma$ elevate IL-4 expression in the ChP and CSF which makes the ChP epithelium to produce CCL11. This chemokine is associated with age-related cognitive dysfunction and shifts the ChP immune phenotype toward destructive T-helper cells type 2 (Th2) (28). This IFN $\gamma$ and IL-4 balance is pivotal for normal CNS immunosurveillance, memory, and cognition physiology at the ChP.

Table 1. CNS-borne factors of immunoregulation

\begin{tabular}{|c|c|c|c|c|c|}
\hline \multicolumn{2}{|c|}{ Mediators } & \multirow{2}{*}{$\begin{array}{c}\text { Location } \\
\text { ChP epithelium }\end{array}$} & \multirow{2}{*}{$\begin{array}{l}\text { Immunoregulation } \\
\text { Leukocytes }^{\#}\end{array}$} & \multirow{2}{*}{$\begin{array}{c}\text { Disease } \\
\begin{array}{c}\text { ChP papillomas and } \\
\text { carcinomas }\end{array}\end{array}$} & \multirow{2}{*}{$\begin{array}{l}\text { Refs. } \\
13\end{array}$} \\
\hline Adhesion molecules & E-cadherin & & & & \\
\hline & $\begin{array}{l}\text { ICAM-1, } \\
\text { VCAM-1, } \\
\text { MAdCAM-1 }\end{array}$ & ChP epithelium & Leukocytes $^{\#}$ & MS & 14 \\
\hline & P-selectin & ChP endothelium & $\mathrm{CD} 4^{+} \mathrm{T}^{\#}$ & MS & 15 \\
\hline \multirow[t]{3}{*}{ Chemokines } & CCL20 & ChP epithelium & Th17 cells & $\begin{array}{l}\text { MS, pneumococcal } \\
\text { meningitis }\end{array}$ & 18,19 \\
\hline & CCL19, CCL21 & ChP epithelium & $\begin{array}{l}\mathrm{CCR7}^{+} \text {central } \\
\text { memory T cells }^{\#}\end{array}$ & Neuroinflammation & 20,21 \\
\hline & CX3CL1 & ChP endothelium & $\begin{array}{l}\text { M2 M } \phi^{\#} \\
\text { Monocytes }\end{array}$ & $\begin{array}{l}\text { Spinal cord injury } \\
\text { Stroke }\end{array}$ & $23-25$ \\
\hline \multirow[t]{4}{*}{$\begin{array}{l}\text { Cytokines/ } \\
\text { neuropeptides }\end{array}$} & $\mathrm{IFN} \gamma$ & ChP epithelium & Leukocytes $^{\#}$ & $\begin{array}{l}\text { Host defense } \\
\text { MS }\end{array}$ & 26,27 \\
\hline & IL-4 & $\mathrm{ChP}$ & *Th2 cells & Aging & 28 \\
\hline & TGF $\beta$ & CSF, ChP & $\begin{array}{l}\downarrow \mathrm{CD}^{+}, \text {Th17 cells; } \\
\uparrow \text { Tregs }\end{array}$ & $\mathrm{AD}, \mathrm{TBI}$ & $29-32$ \\
\hline & $\mathrm{PGD}_{2}$ & CSF & $\begin{array}{l}\text { Neuronal protection } \\
\text { A2- Astrocytes }\end{array}$ & PD & 35,36 \\
\hline
\end{tabular}

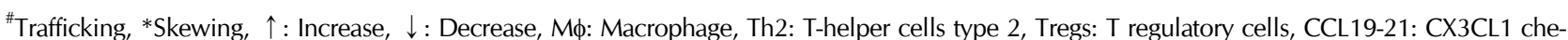
mokines, ChP: Choroid plexus, CSF: Cerebrospinal fluid, ICAM-1: intercellular adhesion molecule-1, VCAM-1: vascular cell adhesion molecule-1, MAdCAM-1: mucosal addressin cell adhesion molecule-1, IFN $\gamma$ : interferon gamma, IL-4: interleukin 4, TGF $\beta$ : transforming growth factor beta, $\mathrm{PGD}_{2}$ : prostaglandin D2, A2- Astrocytes: Anti-inflammatory astrocytes, AD: Alzheimer's disease, MS: Multiple sclerosis, PD: Parkinson's disease, $\mathrm{TBI}$ : traumatic brain injury. 


\section{Immune choreography by CSF}

ChP and CSF are home to several growth factors such as TGF $\beta$, vascular endothelial growth factor (VEGF), and several others. Among these mediators TGF $\beta$ and VEGF have the most pronounced effects; both hold ChP integrity, regulate ChP ECs stability, modulate ependymal cells CSF secretion, and affect BCSFB permeability (29). CSF-residing TGF $\beta$ is anticipated to act during traumatic brain injury by obstructing the cytotoxic $\mathrm{CD}^{+}(30)$ and Th17 cells activity and stimulating Tregs production to reduce inflammation, during which time CSF levels of TGF $\beta$ are maximally increased (31). Indeed, intracerebroventricular and intranasal administration of TGF $\beta$ suppresses glia- and T-cell-induced inflammation and alleviates AD-related neurodegeneration (32).

PGD2 is a bioactive inflammatory lipid mediator found in high concentrations in both CSF and ChP (33). It regulates a broad array of neurophysiological functions such as the sleepwake cycle, body temperature, nociception, and immunomodulation (34). $\mathrm{PGD}_{2}$ rescues hippocampal neurons from glutamate (35) and aluminum cytotoxicity (36) through cyclic adenosine monophosphate and calcium ion signaling by the DP1 receptor. It provides astrocytes with their anti-inflammatory functions and directly modulates the pro-inflammatory roles of microglia in a mouse model of Parkinson's disease (PD) (37). Altogether, these findings suggest the extraordinary potential of ChP epithelium and endothelium to express adhesion molecules, chemokines, and cytokines which are pivotal for the generation of an immune cell homing niche in the CNS (Table 1).

\section{IMMUNOPATHOLOGY: THE ChP-CSF SYSTEM IN DISEASE}

BCSFB impairment is seen in neurodegenerative disorders, including $A D$, as well as neuroinflammatory diseases, such as multiple sclerosis (MS) and major depressive disorder (MDD), and physiological aging causes dysfunctions in the ChP-CSF system. Understanding the BCSFB during CNS abnormalities could open new avenues to explore brain pathology and how to treat it.

\section{MS}

MS is an autoimmune disease characterized by chronic inflammation, demyelination, and degeneration of CNS neurons. With the help of a well-established animal model (i.e., the EAE model), the etiology of MS in the context of immunopathology has been extensively studied. Studies of the EAE model and MS patients have confirmed the overwhelming presence and necessity of CCR6-expressing pathogenic Th17 cells that enter the CNS parenchyma through the CCL20-expressing ChP epithelial cells in establishment of the disease (Fig. 2C) $(18,38)$.

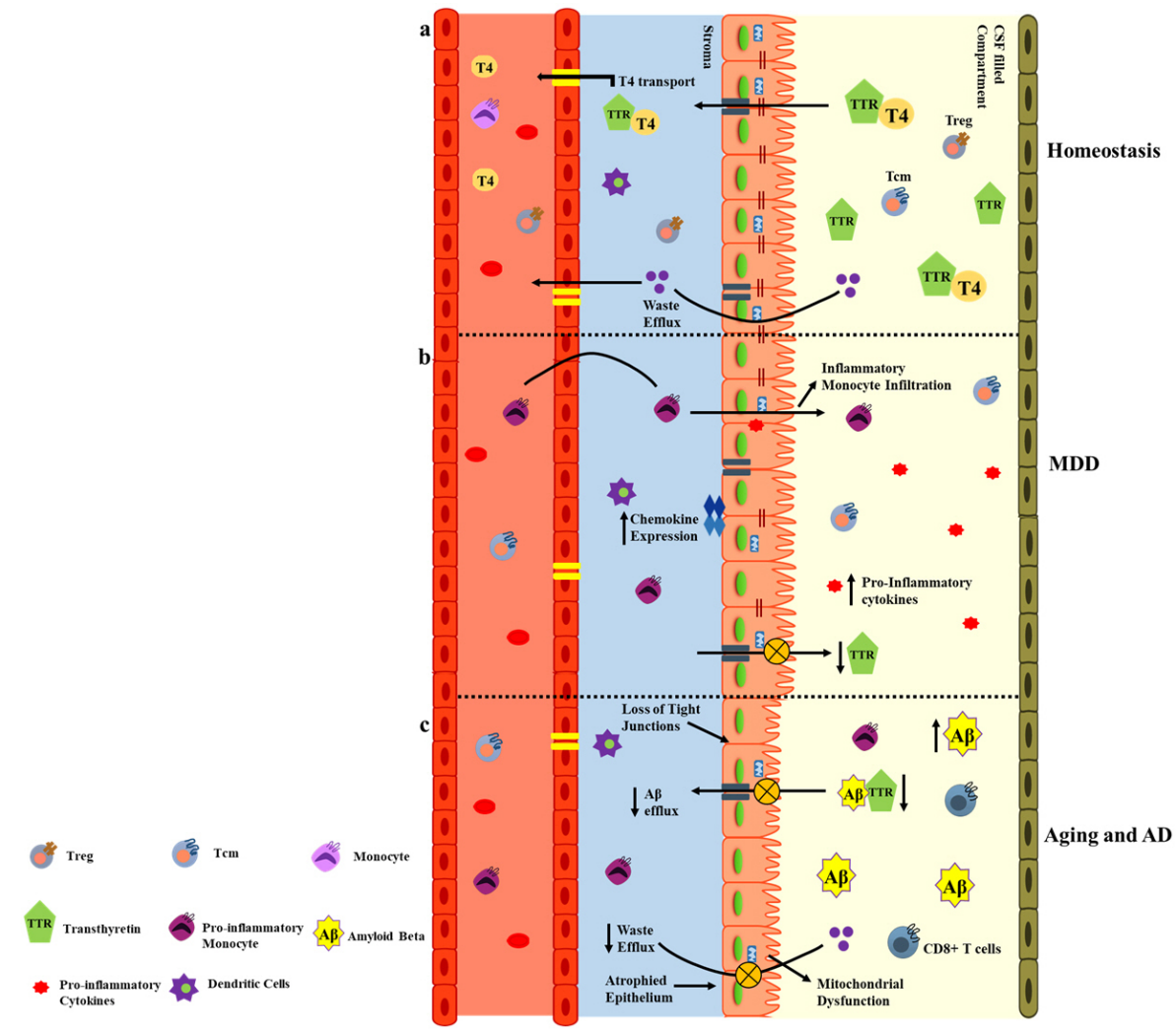

Fig. 3. Immunopathology at BCSFB. (A) The homeostatic BCSFB expresses TTR, a protein involved in the transport of thyroid hormones and $A \beta$ to the peripheral circulation. The BCSFB is also populated with mitochondria which support this active transport. (B) In MDD, the ChP tight junctions are partially lost, the TTR levels decrease, and chemokine and pro-inflammatory expression increases causing neuroinflammation. (C) In aging or $\mathrm{AD}$, the $\mathrm{ChP}$ epithelium is atrophied, the TTR levels severely decline which increases $A \beta$ deposition, decreases metabolic waste efflux, disturbs mitochondrial energetics, and intensifies the infiltration of $\mathrm{CD}^{+}{ }^{+}$cells and pro-inflammatory monocytes from the periphery to the CNS. 
Postmortem studies of human MS brain tissues have described the selective loss of the tight junction protein claudin 3 (CLDN3), which is essential for BCSFB function. Loss of this protein enhances leukocyte infiltration into the CSF and brain parenchyma (39). Given the anatomy and molecular machinery of the ChP, it is evident that the ChP is essential in the initiation and development of MS.

\section{MDD}

MDD is a debilitating condition affecting 264 million people and is a leading cause of disability worldwide. The pathogenesis of MDD in the context of immunopathology has emerged in recent years and the ChP-CSF system has gained much attention in this context. One study has reported the compensatory role of the ChP in depressive suicidal patients; indeed, IL-1 $\beta$, ICAM-1, and microglia expression (Iba-1) were downregulated in the ChP of depressive suicidal subjects (40). The synthesis of transthyretin (TTR), a transporter protein of the $\mathrm{ChP}$, is regulated by serotonin. Patients suffering from neuropsychiatric disorders have inadequate $5-\mathrm{HT}_{2 \mathrm{C}}$ (serotonin receptors) receptor activation due to reduced serotonin expression, and therefore low levels of TTR (Fig. 3A, B) (41). The altered functionality of the ChP and CSF secretome indicates that the ChP-CSF system could be utilized for the early diagnosis and treatment of MDD.

\section{Aging and AD}

Recent studies have recognized morphological and molecular changes in the BCSFB and disrupted CSF production and turnover during aging and neurodegenerative disorders, especially AD. During physiological aging the $\mathrm{ChP}^{\prime} \mathrm{s}$ cuboidal epithelium flattens, the machinery for oxidative phosphorylation and anaerobic respiration is diminished, the CSF secretion and turnover rate is altered, and the barrier permeability is jeopardized (42). During $A D$, the ChP-CSF system transport of $A \beta$ plaque from the brain via TTR is disrupted. Decreased TTR levels in the brains of $A D$ patients increase $A \beta$ deposition (43). Both in vitro and in vivo studies of $\mathrm{AD}$ models have observed elevated levels of nitric oxide in the AD brain. This results in NF-kB signaling retention, which reduces the expression of IFN $\gamma \mathrm{R}$ on the ChP epithelium. Since IFN $\gamma \mathrm{R}$ aids leukocyte trafficking at the ChP, its reduction exacerbates the neurodegeneration (Fig. 3C) (44). Altogether, changes in $\mathrm{ChP}$ morphology and physiology along with CSF alterations contribute to the $\mathrm{AD}$ and aging pathology.

\section{CONCLUDING REMARKS AND FUTURE DIRECTIONS}

This manuscript describes the immunomodulatory gating and barrier strategies of the CNS true versus educational barriers. These barriers work in coordination to guard the CNS against peripheral changes and they provide lines of defense following an injury. These well-regulated barriers are important for appropriate neuronal and cognitive functions, but the restrictive nature of true barriers poses therapeutic challenges, in drug delivery and lowers rates of recovery. Thus, neuromedicine mainly focuses on identifying strategies to improve targeted drug delivery, and manipulating the properties of the ChP-CSF system because of its functional relevance to the BCSFB has promising applications in neurotherapies. Indeed, further characterization of the BCSFB's molecular constituents could help researchers determine the usefulness of this system as a therapeutic target for neurological disorders.

\section{ACKNOWLEDGEMENTS}

This research was supported by the Basic Science Research Program (2017R1A4A1015652, 2020R1A2C3006875, 2020R $1 \mathrm{~A} 2 \mathrm{C} 3006734)$ of the NRF funded by the Korean government, MSIT.

\section{CONFLICTS OF INTEREST}

The authors have no conflicting interests.

\section{REFERENCES}

1. Murphy JB and Sturm E (1923) Conditions determining the transplantability of tissues in the brain. J Exp Med 38, 183-197

2. Galea I, Bechmann I and Perry VH (2007) What is immune privilege (not)? Trends Immunol 28, 12-18

3. Louveau A, Smirnov I, Keyes TJ et al (2015) Structural and functional features of central nervous system lymphatic vessels. Nature 523, 337-341

4. Iliff JJ, Lee H, Yu M et al (2013) Brain-wide pathway for waste clearance captured by contrast-enhanced MRI. J Clin Invest 123, 1299-1309

5. Alvarez JI, Dodelet-Devillers A, Kebir H et al (2011) The hedgehog pathway promotes blood-brain barrier integrity and CNS immune quiescence. Science 334, 1727-1731

6. McCandless EE, Wang Q, Woerner BM, Harper JM and Klein RS (2006) CXCL12 limits inflammation by localizing mononuclear infiltrates to the perivascular space during experimental autoimmune encephalomyelitis. J Immunol 177, 8053-8064

7. Ifergan I, Kébir H, Bernard M et al (2008) The blood-brain barrier induces differentiation of migrating monocytes into Th17-polarizing dendritic cells. Brain 131, 785-799

8. Weller RO (2005) Microscopic morphology and histology of the human meninges. Morphologie 89, 22-34

9. Bartholomäus I, Kawakami N, Odoardi F et al (2009) Effector $\mathrm{T}$ cell interactions with meningeal vascular structures in nascent autoimmune CNS lesions. Nature 462, 94-98

10. Meeker RB, Williams K, Killebrew DA and Hudson LC (2012) Cell trafficking through the choroid plexus. Cell Adh Migr 6, 390-396

11. Marques F, Sousa JC, Brito MA et al (2017) The choroid plexus in health and in disease: dialogues into and out of the brain. Neurobiol Dis 107, 32-40

12. Harjunpää $\mathrm{H}$, Llort Asens $M$, Guenther $C$ and Fagerholm SC (2019) Cell adhesion molecules and their roles and regulation in the immune and tumor microenvironment. Front Immunol 10, 1078

13. Figarella-Branger D, Lepidi $H$, Poncet C et al (1995) 
Differential expression of cell adhesion molecules (CAM), neural CAM and epithelial cadherin in ependymomas and choroid plexus tumors. Acta Neuropathol 89, 248-257

14. Wolburg K, Gerhardt $\mathrm{H}$, Schulz $\mathrm{M}$, Wolburg $\mathrm{H}$ and Engelhardt B (1999) Ultrastructural localization of adhesion molecules in the healthy and inflamed choroid plexus of the mouse. Cell Tissue Res 296, 259-269

15. Kivisäkk P, Mahad DJ, Callahan MK et al (2003) Human cerebrospinal fluid central memory CD4 + T cells: evidence for trafficking through choroid plexus and meninges via P-selectin. Proc Natl Acad Sci U S A 100, 8389-8394

16. Greaves DR, Wang W, Dairaghi DJ et al (1997) CCR6, a CC chemokine receptor that interacts with macrophage inflammatory protein $3 \alpha$ and is highly expressed in human dendritic cells. J Exp Med 186, 837-844

17. Liao F, Rabin RL, Smith CS, Sharma G, Nutman TB and Farber JM (1999) CC-chemokine receptor 6 is expressed on diverse memory subsets of $\mathrm{T}$ cells and determines responsiveness to macrophage inflammatory protein 3 alpha. J Immunol 162, 186-194

18. Reboldi A, Coisne C, Baumjohann D et al (2009) C-C chemokine receptor 6-regulated entry of $\mathrm{TH}-17$ cells into the CNS through the choroid plexus is required for the initiation of EAE. Nat Immunol 10, 514-523

19. Klein M, Brouwer MC, Angele B et al (2014) Leukocyte attraction by CCL20 and its receptor CCR6 in humans and mice with pneumococcal meningitis. PLoS One 9, e93057

20. Krumbholz M, Theil D, Steinmeyer F et al (2007) CCL19 is constitutively expressed in the CNS, up-regulated in neuroinflammation, active and also inactive multiple sclerosis lesions. J Neuroimmunol 190, 72-79

21. Kivisäkk P, Mahad DJ, Callahan MK et al (2004) Expression of CCR7 in multiple sclerosis: implications for CNS immunity. Ann Neurol 55, 627-638

22. Imai T, Hieshima K, Haskell C et al (1997) Identification and molecular characterization of fractalkine receptor CX3CR1, which mediates both leukocyte migration and adhesion. Cell 91, 521-530

23. Pan Y, Lloyd C, Zhou H et al (1997) Neurotactin, a membrane-anchored chemokine upregulated in brain inflammation. Nature 387, 611-617

24. Shechter R, Miller O, Yovel G et al (2013) Recruitment of beneficial M2 macrophages to injured spinal cord is orchestrated by remote brain choroid plexus. Immunity $38,555-569$

25. Ge R, Tornero D, Hirota M et al (2017) Choroid plexuscerebrospinal fluid route for monocyte-derived macrophages after stroke. J Neuroinflammation 14, 153

26. Adam RA, Tenenbaum T, Valentin-Weigand $P$ et al (2004) Porcine choroid plexus epithelial cells induce Streptococcus suis bacteriostasis in vitro. Infect Immun 72, 3084-3087

27. Lee E, Chanamara S, Pleasure D and Soulika AM (2012) IFN-gamma signaling in the central nervous system controls the course of experimental autoimmune encephalomyelitis independently of the localization and composition of inflammatory foci. J Neuroinflammation 9, 7

28. Baruch K, Ron-Harel N, Gal H et al (2013) CNS-specific immunity at the choroid plexus shifts toward destructive Th2 inflammation in brain aging. Proc Natl Acad Sci U S A 110, 2264-2269
29. Maharaj AS, Walshe TE, Saint-Geniez M et al (2008) VEGF and TGF-beta are required for the maintenance of the choroid plexus and ependyma. J Exp Med 205, 491-501

30. Gordon LB, Nolan SC, Ksander BR, Knopf PM and Harling-Berg CJ (1998) Normal cerebrospinal fluid suppresses the in vitro development of cytotoxic T cells: role of the brain microenvironment in CNS immune regulation. J Neuroimmunol 88, 77-84

31. Morganti-Kossmann MC, Hans $\mathrm{VH}$, Lenzlinger $\mathrm{PM}$ et al (1999) TGF-beta is elevated in the CSF of patients with severe traumatic brain injuries and parallels blood-brain barrier function. J Neurotrauma 16, 617-628

32. Chen JH, Ke KF, Lu JH, Qiu YH and Peng YP (2015) Protection of TGF- $\beta 1$ against neuroinflammation and neurodegeneration in A $\beta 1$-42-induced Alzheimer's disease model rats. PLoS One 10, e0116549

33. Pentreath VW, Rees K, Owolabi OA, Philip KA and Doua F (1990) The somnogenic T lymphocyte suppressor prostaglandin D2 is selectively elevated in cerebrospinal fluid of advanced sleeping sickness patients. Trans R Soc Trop Med Hyg 84, 795-799

34. Harizi H (2013) The immunobiology of prostanoid receptor signaling in connecting innate and adaptive immunity. Biomed Res Int 2013, 683405

35. Liang X, Wu L, Hand T and Andreasson K (2005) Prostaglandin D2 mediates neuronal protection via the DP1 receptor. J Neurochem 92, 477-486

36. Ma J, Yang Q, Wei Y et al (2016) Effect of the PGD2-DP signaling pathway on primary cultured rat hippocampal neuron injury caused by aluminum overload. Sci Rep 6, 24646

37. Choi DJ, An J, Jou I, Park SM and Joe EH (2019) A Parkinson's disease gene, DJ-1, regulates anti-inflammatory roles of astrocytes through prostaglandin $\mathrm{D}(2)$ synthase expression. Neurobiol Dis 127, 482-491

38. Komiyama Y, Nakae S, Matsuki T et al (2006) IL-17 plays an important role in the development of experimental autoimmune encephalomyelitis. J Immunol 177, 566-573

39. Kooij G, Kopplin K, Blasig R et al (2014) Disturbed function of the blood-cerebrospinal fluid barrier aggravates neuro-inflammation. Acta Neuropathol 128, 267-277

40. Devorak J, Torres-Platas SG, Davoli MA, Prud'homme J, Turecki G and Mechawar N (2015) Cellular and molecular inflammatory profile of the choroid plexus in depression and suicide. Front Psychiatry 6, 138

41. Sullivan GM, Mann JJ, Oquendo MA, Lo ES, Cooper TB and Gorman JM (2006) Low cerebrospinal fluid transthyretin levels in depression: correlations with suicidal ideation and low serotonin function. Biol Psychiatry 60, 500-506

42. Preston JE (2001) Ageing choroid plexus-cerebrospinal fluid system. Microsc Res Tech 52, 31-37

43. Chen RL, Athauda SB, Kassem NA, Zhang Y, Segal MB and Preston JE (2005) Decrease of transthyretin synthesis at the blood-cerebrospinal fluid barrier of old sheep. J Gerontol A Biol Sci Med Sci 60, 852-858

44. Baruch K, Kertser A, Porat Z and Schwartz M (2015) Cerebral nitric oxide represses choroid plexus NFkappaBdependent gateway activity for leukocyte trafficking. EMBO J 34, 1816-1828 